\title{
artigo
}

\section{Educação permanente na Triagem Neonatal: uma revisão integrativa}

\author{
Permanent education in Neonatal Screening: an integrative review \\ Educación permanente en cribado neonatal: una revisión integradora
}

\begin{abstract}
RESUMO
Objetivo: Investigar estudos sobre educação/capacitação permanente relacionados a triagem neonatal (TN) e/ou teste do pezinho, identificando estratégias que favoreçam futuras ações relativas ao tema. Métodos: Trata-se de um estudo descritivo por meio de revisão integrativa, realizado em agosto de 2019, nas bases de dados "LILACS", "MEDLINE", "IBECS" e "SciELO", através dos termos: "Triagem Neonatal", "Teste do Pezinho", "Capacitação" e "Educação em Saúde". Resultados: A amostra final foi composta por 12 estudos, a maioria da base de dados Medline $(n=11)$. Dentre os artigos analisados, três foram publicados em periódicos nacionais e nove em periódicos internacionais. Considerando as modalidades de TN, sete estudos abordaram a TN biológica, enquanto as triagens auditiva e de cardiopatias foram referidas por quatro e um artigos, respectivamente. A população mais estudada foi de enfermeiros e pais. Conclusão: Ratifica-se a importância de implantar programas de educação permanente e capacitação, abordando estratégias eficazes para melhorar a Triagem Neonatal Universal.
\end{abstract}

DESCRITORES: Educação Permanente; Triagem Neonatal; Saúde Infantil.

\section{ABSTRACT}

Objective: To investigate studies on education / permanent training related to neonatal screening (NS), identifying strategies that favor future actions related to the theme. Methods: This is a descriptive study through an integrative review, conducted in August 2019, in the databases "LILACS", "MEDLINE", "IBECS" and "SciELO", through the terms: "Triagem Neonatal", "Teste do Pezinho", "Capacitação" and "Educação em Saúde". Results: The final sample consisted of 12 studies, the majority from the Medline database $(n=11)$. Among the articles analyzed, three were published in national journals and nine in international journals. Considering the modalities of NS, seven studies addressed biological NS, while hearing and heart screening were mentioned by four and one articles, respectively. The most studied population was nurses and parents. Conclusion: The importance of implementing permanent education and training programs is ratified, addressing effective strategies for the improvement of Universal Neonatal Screening. DESCRIPTORS: Permanent Education; Neonatal Screening; Children Health.

\section{RESUMEN}

Objetivo: Investigar estudios sobre educación / formación permanente relacionados con el cribado neonatal, identificando estrategias que favorezcan acciones futuras relacionadas con la temática. Métodos: Se trata de un estudio descriptivo mediante revisión integradora, realizada en agosto de 2019, en las bases de datos "LILACS", "MEDLINE", "IBECS" y "SciELO", através de los términos: "Triagem Neonatal", "Teste do Pezinho", "Capacitação" y "Educação em Saúde". Resultados: La muestra final consistió en 12 estudios, la mayoría de la base de datos Medline $(n=11)$. Entre los artículos analizados, tres fueron publicados en revistas nacionales y nueve en revistas internacionales. Teniendo en cuenta las modalidades de la cribado neonatal, siete estudios abordaron la cribado neonatal biológica, mientras que cuatro y un artículo mencionaron la detección auditiva y cardíaca, respectivamente. La población más estudiada fueron enfermeras y padres. Conclusión: Se ratifica la importancia de implementar programas de educación y capacitación permanente, abordando estrategias efectivas para el mejoramiento del Cribado Neonatal Universal.

DESCRIPTORES: Educación Permanente; Cribado neonatal; Salud infantil.

RECEBIDO EM: 28/11/2020 APROVADO EM: 24/12/2021

\section{Amanda Borges Ferreira}

Discente no Curso de Farmácia, Universidade Federal de Mato Grosso (UFMT).

ORCID: 0000-0001-7799-2341 


\section{Maria Fernanda Spegiorin Salla Brune}

Farmacêutica, Doutora, Docente no Curso de Farmácia na Universidade Federal de Mato Grosso (UFMT). ORCID: 0000-0002-1822-0904

\section{INTRODUÇÃO}

A Triagem Neonatal (TN) é um programa de rastreamento populacional que tem como objetivo identificar distúrbios e doenças no recém-nascido, garantindo tratamento e acompanhamento contínuo àqueles com diagnóstico positivo. No Brasil, este programa é amparado pela Portaria GM/MS no 822 , de 6 de junho de 2001, e realizado nas modalidades biológica, auditiva, ocular e de cardiopatias congênitas críticas, além da avaliação do frênulo lingual. A TN biológica, popularmente denominado "teste do pezinho", utiliza como estratégia exames laboratoriais para identificar possíveis doenças no neonato, sendo disponível no Sistema Único de Saúde o diagnóstico de Fenilcetonúria, Fibrose Cística, Hiperplasia Adrenal Congênita, Deficiência de Biotinidase, Anemia Falciforme e Hipotireoidismo Congênito ${ }^{(1,2,3)}$.

O alcance de uma cobertura de 100\% dos recém-nascidos no Programa Nacional de Triagem Neonatal é dificultado por vários fatores que dificultam o acesso ao teste, destacando-se as falhas na orientação durante o pré-natal por parte dos profissionais de saúde $e^{(4,5)}$.

$\mathrm{O}$ desenvolvimento de estratégias para a educação de pais sobre TN tem sido um desafio, gerando incertezas sobre o conteúdo apropriado a ser apresentado a este público. Neste contexto, destaca-se ainda a importância do profissional da saúde na interação com os pais, estabelecendo uma sensação de segurança e orientando-os no sentindo de promover apoio à saúde do neonato ${ }^{(6,7)}$.

Pesquisas têm apontado para um conhecimento insuficiente sobre triagem neonatal por parte da equipe de saúde. Essa lacuna poderia ser preenchida com ações de educação permanente e continuada. Todavia, apesar da relevância do assunto, há escassez de produções científicas que tratem da atuação e promoção educacional de equipes de saúde em relação à $\mathrm{TN}^{(8,9)}$.
Esta pesquisa teve como objetivo investigar estudos sobre educação/capacitação em saúde relacionados a triagem neonatal e/ou teste do pezinho, a fim de identificar estratégias que possam favorecer o desenvolvimento de futuras ações educativas relativas ao tema.

\section{MÉTODOS}

Trata-se de um estudo descritivo de revisão integrativa, elaborada em seis etapas: (1) identificação do tema e seleção de hipótese; (2) estabelecimento de critérios para inclusão e exclusão de estudos e escolha da amostra; (3) definição das informações a serem extraídas dos artigos selecionados; (4) avaliação dos estudos inseridos na revisão integrativa; (5) interpretação dos resultados e discussões; (6) síntese e apresentação da revisão ${ }^{(10)}$.

A pesquisa dos artigos foi realizada em agosto de 2019. Foi utilizada como seleção estratégica de buscas de artigos os termos: "Triagem Neonatal", "Teste do Pezinho", "Capacitação" e "Educação em Saúde", com a intenção de englobar o tema, permitindo uma ampla busca, porém, de forma direcionada.
Os critérios de inclusão definidos para a seleção foram: (a) artigo original, publicado nos idiomas português, inglês ou espanhol, (b) entre 2009 e 2019, nas bases de dados "LILACS", "MEDLINE", "IBECS" e "SciELO". Para serem incluídos, os estudos deveriam ter desenvolvido alguma ação de educação em saúde e/ou capacitação, abordando o tema triagem neonatal e/ou teste do pezinho. Foram excluídas as dissertações e teses, publicações referentes a resumos de congressos, anais, editoriais, comentários e opiniões, artigos de reflexão, projetos, relatórios e informes técnicos.

A escolha dos artigos foi feita em quatro etapas, ilustradas na Figura 1: fase de identificação, em que foram pesquisados os termos estratégicos; fase de seleção, em que foram utilizados critérios para inclusão e exclusão; fase de elegibilidade, contendo pressupostos necessários para a escolha dos artigos; e a fase de inclusão, a qual definiu quais artigos seriam inseridos no estudo.

Esta revisão incluiu 12 estudos, apresentados no Quadro 1, sendo 11 artigos encontrados na base de dados "Medline" e 1 identificado na base de dados "SciELO". Dentre os 12 artigos analisados, três foram publicados
Figura 1. Fluxograma de identificação e seleção dos artigos para a revisão integrativa na abordagem da educação continuada e capacitação sobre triagem neonatal.

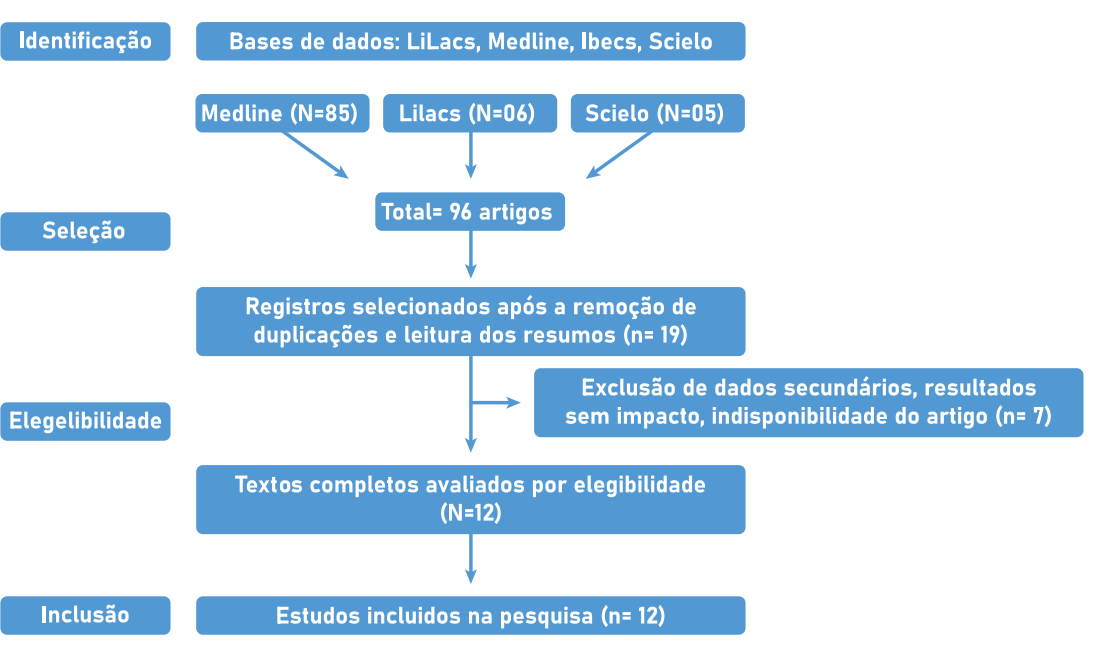




\section{artigo}

em periódicos nacionais, escritos na língua portuguesa, e nove foram publicados em periódicos internacionais, sendo oito destes na língua inglesa e um na língua portuguesa.

\section{RESULTADOS E DISCUSSÕES}

A Triagem Neonatal Universal encontra-se inserida na Política Nacional de Atenção Integral à Saúde da Criança (PNAISC) (Portaria GM/MS n. ${ }^{\circ} 1.130$ de 5 de agosto de 2015) descritas no Eixo Estratégico 1, cujo objetivo é promover e proteger a saúde da criança e o aleitamento materno. Para isso, de acordo com o Ministério da Saúde, são necessários recursos humanos disponíveis e em constante processo de educação permanente, para acesso articulado e oportuno em todos os níveis de atenção considerando o cuidado individual, de educação e ações coletivas de saúde ${ }^{(2)}$.

Nesta revisão integrativa foram selecionados artigos relacionados à importância da educação em saúde na abordagem da triagem neonatal, com enfoques na educação permanente e na capacitação em saúde.
Dentre os 12 artigos analisados nesta revisão, sete estudos abordaram a TN biológica ${ }^{(7,11-16)}$, enquanto a triagem auditiva foi referida por quatro artigos ${ }^{(10,17,18,20)} \mathrm{e}$ apenas um artigo apresentou dados sobre a TN de cardiopatias ${ }^{(19)}$.

Nos estudos 1, 4, 5, 6, 7, 8 e $10^{(7,11-16)}$ (Tabela 1) foi abordada a importância da educação na saúde em relação a triagem biológica, sob diferentes perspectivas.

Nos artigos 1,5 e $8^{(11,12,15)}$ a educação na saúde foi direcionada para o diagnóstico de fibrose cística, enquanto no artigo 6 ${ }^{(13)} \mathrm{o}$ enfoque foi para a doença falciforme, ambas diagnosticadas pelo "teste do pezinho". Na pesquisa realizada no estudo 8 (15), todas as 12 famílias entrevistadas relataram terem recebido informações sobre TN durante a gravidez ou logo após o nascimento, verbalmente ou por escrito. No entanto, os pais relataram não terem lido o material sobre TN disponibilizado, pois sentiram-se falsamente tranquilizados pelos profissionais de saúde, os quais informaram sobre a baixa probabilidade de um resultado positivo. A informação apresen- tada neste estudo evidencia que a educação continuada sobre TN, direcionada aos pais, não é padronizada e isso gera informaçóes imprecisas e subjetivas da própria equipe de saúde.

A dificuldade na comunicação entre pais e equipe de saúde sobre a TN também foi discutida nos estudos 1,5 e $6^{(11-13)}$, em que os pais enfatizam a necessidade de melhorar a comunicação sobre TN durante o período perinatal, e sugerem o esclarecimento do objetivo da TN e quais doenças são incluídas no exame.

A falta de conhecimento sobre TN tem um impacto, inclusive, na expectativa de vida de crianças com diagnóstico positivo na TN. A pesquisa do artigo $6^{(13)}$ analisou a ocorrência de óbitos em crianças com doença falciforme, e concluiu que o déficit de conhecimento sobre a doença pelos profissionais de saúde foram fatores agravantes do quadro clínico dos pacientes.

No Brasil, documentos do Ministério da Saúde publicados em 2016 e 2018 apenas citam que as informaçóes sobre TN devem ser levadas aos pais pela equipe de

Quadro 1- Descrição dos artigos incluídos na revisão integrativa na abordagem da educação continuada e capacitação sobre triagem neonatal.

\begin{tabular}{|c|c|c|c|}
\hline No & PERIÓDICO/TIPO/ANO & OBJETIVOS & TIPO DE ESTUDO \\
\hline 1 & J Perinat Neonat Nurs 2009 $9^{(11)}$ & Investigar oferta de informações sobre TN aos pais & Qualitativo \\
\hline 2 & Adv in Neonatal Care $2014^{(19)}$ & $\begin{array}{l}\text { Avaliar o conhecimento dos enfermeiros sobre a } \\
\text { triagem da doença cardíaca congênita }\end{array}$ & Quase experimental \\
\hline 3 & Revista CEFAC 2014(10) & $\begin{array}{l}\text { Avaliar o conhecimento dos enfermeiros em ações } \\
\text { educativas em saúde auditiva infantil. }\end{array}$ & Revisão integrativa \\
\hline 4 & J Genetic Counsel $2015^{(7)}$ & $\begin{array}{l}\text { Explorar os modelos de educação em saúde direciona- } \\
\qquad \text { da aos pais. }\end{array}$ & Revisão sistemática \\
\hline 5 & J Genetic Counsel $2015^{(12)}$ & Avaliar o conhecimento dos pais sobre a fibrose cística & Prospectivo e randomizado \\
\hline 6 & Jornal de Pediatria $2015^{(13)}$ & Caracterizar óbitos de crianças com doença falciforme & Pesquisa clínica e epidemiológica. \\
\hline 7 & JAMA Pediatrics 2016(14) & $\begin{array}{c}\text { Analisar o efeito da educação pré-natal sobre triagem } \\
\text { neonatal }\end{array}$ & Ensaio clínico randomizado \\
\hline 8 & J Genet Counsel 2016(15) & $\begin{array}{l}\text { Explorar as experiências dos pais após resultado positi- } \\
\text { vo para fibrose cística ou doença falciforme. }\end{array}$ & Qualitativo \\
\hline 9 & J Midwifery Womens Health 2016(17) & $\begin{array}{l}\text { Analisar a abordagem da triagem auditiva no currículos } \\
\text { de medicina }\end{array}$ & Levantamento de dados \\
\hline 10 & Rev. Paul. Pediatr. 2017(16) & $\begin{array}{l}\text { Avaliar o conhecimento das puérperas sobre o teste do } \\
\text { pezinho. }\end{array}$ & Descritivo e quantitativo \\
\hline 11 & Int J Pediatr Otorhinolaryngol 2017(20) & Avaliar a oferta de informações sobre TN aos pais & Levantamento de dados \\
\hline 12 & Braz J Otorhinolaryngol 2013 ${ }^{(18)}$ & Investigar estudos sobre educação em triagem auditiva & Quase experimental \\
\hline
\end{tabular}


saúde, sem nenhum detalhe sobre como e quando realizar esta abordagem, nem sobre informações essenciais que deveriam ser consideradas ${ }^{(1,2)}$. Em tempo, é importante reforçar que as ações de educação em saúde apontam para três segmentos de atores prioritários: os profissionais de saúde, atuando na prevenção de doenças, na promoção de saúde e nas práticas curativas; os gestores, fornecendo apoio a esses profissionais; e a população que necessita construir seus conhecimentos e aumentar sua autonomia nos cuidados, individual e coletivamente $^{(21)}$.

A pesquisa feita no estudo $5^{(12)}$ concluiu que vídeos educativos sobre TN podem, além de aumentar a adesão dos pais a efetuarem o teste, melhorar a compreensão e reduzir a ansiedade de pais que possam receber um resultado positivo no exame da TN.

$\mathrm{O}$ artigo $10^{(16)}$ concluiu que o conhecimento das puérperas sobre a TN é superficial e pode ser reflexo da fragilidade de atuação da equipe de saúde, e enfatizou a necessidade de se priorizar ações de educação permanente nos serviços de saúde que tenham como foco a TN.

É necessário capacitar os profissionais envolvidos, avaliar a disseminação de informação e avaliar tais intervenções educativas, a fim de documentar as necessidades locais de triagem neonatal e os entraves deste processo, para posterior adoção de estratégias ${ }^{(10)}$. Cabe ressaltar que, se considerarmos a TN biológica, a qual envolve o diagnóstico de seis doenças, cada qual com sua patologia e consequências distintas e complexas, é notória a dificuldade em elaborar um material conciso e de fácil compreensão.

O autor do artigo $9^{(17)}$ entrevistou diretores de Programas de Educação em Obstetrícia nos EUA (Programa de Residência Médica) e, apesar de todos os entrevistados confirmarem a inclusão da TN auditiva no currículo, nota-se que questões mais profundas sobre $\mathrm{TN}$ não são debatidas, como o aconselhamento dos pais sobre possíveis resultados positivos e o acompanhamento de crianças não triadas ao nascer.
Outros estudos sobre TN auditiva $(10,18,20)$ também apontam para a necessidade de informações mais precisas sobre o assunto aos pais. Em uma revisão integrativa sobre TN auditiva, o estudo $3^{(10)}$ afirma a necessidade de ampliar o conhecimento dos profissionais da saúde através de educação permanente.

Propostas de ações educativas aos profissionais de saúde também foram apresentadas por outros estudos ${ }^{(10,18,19)}$. Nos artigos 3 e $12^{(10,18)}$, os autores realizaram ações educativas voltadas para a saúde auditiva infantil e as consideraram positivas no sentido de modificar o conhecimento dos profissionais.

A necessidade de que as instituições de saúde forneçam instrução e educação suficientes antes da implementação de novos protocolos ou mudanças na prática clínica também ficou evidenciado no estudo $2^{(19)}$, ao avaliar o quanto a educação online de enfermeiros foi eficiente no diagnóstico de doenças cardíacas na TN.

As práticas de educação em saúde são inerentes ao trabalho em saúde, mas muitas vezes estão relegadas a um segundo plano na organização dos serviços e na própria gestão ${ }^{(21)}$. Este cenário não é exclusivo do Brasil, em que a educação em TN muitas vezes não é priorizada, com orçamentos baixos ou inexistentes, exigindo que os programas sejam eficazes com pouco financiamento. Neste contexto, pesquisadores norte-americanos desenvolveram um documento intitulado "Modelo de Melhores Práticas de Educação para Triagem Neonatal", no qual um fluxograma auxilia na identificação das dificuldades envolvidas no processo da triagem neonatal, e também aponta para questões práticas na implementação de ações educativas, bem como sua viabilidade em diferentes situações. Segundo os autores, o compartilhamento de estratégias de sucesso entre programas de TN mitiga a escassez de recursos e reduz a carga de trabalho ${ }^{(22)}$.

A pesquisa do artigo $4^{(7)}$ discute quatro diferentes modelos de Programas de Triagem Neonatal em diferentes países, e mostra seus impactos no conhecimento dos pais sobre o tema. De acordo com os autores, atender às expectativas e preferências dos pais sobre tipos de abordagens para implementar a educação em saúde é um objetivo importante, e apontam que o tipo de educação continuada a ser ofertada depende do grau de envolvimento dos pais frente a decisão de participar ou não da TN.

A importância da adequação do material a ser utilizado na educação continuada também mostrou-se evidente nos trabalhos 3 e $4^{(7,10)}$. Por isso, a investigação a quem será destinado o material educativo, tendo como parâmetros fatores socioeconômicos e demográficos, é fundamental para a preservação da inteligibilidade e facilidade do uso do material ${ }^{(10)}$.

Ao constatar as dificuldades no monitoramento de ações implementadas na TN auditiva, um Modelo Lógico do Programa de TN auditiva foi elaborado e validado no Brasi ${ }^{(23)}$. O Modelo Lógico é definido como um esquema visual que facilita o planejamento de ações e o gerenciamento de um programa, e neste caso a educação em triagem auditiva foi uma das dimensões trabalhadas. Corroborando com estudos anteriores $^{(12,18)}$, foram sugeridas ações de orientação por meio de rodas de conversa nos ambientes onde pais e profissionais de saúde estão habitualmente inseridos: salas de espera, reunióes clinicas, dentre outros, a fim de que a troca de informações seja mais eficaz. Esta abordagem vislumbra a corresponsabilização dos pais, já que um dos maiores entraves ao sucesso da TN auditiva, segundo os autores, é justamente a não adesão dos pais e familiares aos referenciamentos realizados ${ }^{(23)}$.

$\mathrm{Na}$ pesquisa do estudo $7^{(14)}$, realizada nos Estados Unidos (EUA), o conhecimento e atitudes dos pais em relação à TN teve um enfoque específico além da TN: a retenção e uso de material biológico do recém-nascido para pesquisa. Um aspecto interessante citado nesta pesquisa é de que o agito pós-parto pode contribuir para baixas eficácia das atuais abordagens educacionais realizadas nesta fase tardia.

Por fim, é enfatizado a necessidade de mais pesquisas para avaliar as melhores práticas para implementação da educação em TN durante a prática obstétrica de ro- 


\section{artigo}

tina. Segundo dados de um relatório técnico que sintetiza a situação do PNTN, publicado em 2013, as atividades educativas restringem-se, na maioria dos estados, à capacitação dos técnicos responsáveis pela coleta de amostras. Neste sentido, o documento aponta que as ações de educação permanente em $\mathrm{TN}$ podem contar com recursos específicos transferidos pelo
MS para este fim, pleiteados nos espaços de negociação bipartite (Comissão Intergestora Bipartite e Comissão Intergestora Regional), passando a integrar os Planos Estaduais de Educação Permanente ${ }^{(2)}$.

\section{CONCLUSÃO}

Nesta revisão integrativa, constatou-se uma carência na literatura nacional e internacional se tratando de programas de educação em saúde na TN, especialmente para profissionais da saúde. Foram revisados métodos efetivos para nortear futuras elaborações de programas de educação permanente na $\mathrm{TN}$, apresentando intervenções através de ações educativas com sugestôes concretas e atualizadas. -

\section{REFERÊNCIAS}

1. Brasil. Ministério da Saúde. Secretaria de Atenção a Saúde. Departamento de Atenção Especializada e Temática. Triagem neonatal biológica: manual técnico. Brasília: Ministério da Saúde, 2016. 80 p.: il.

2. Núcleo de Ações e Pesquisa em Apoio Diagnóstico-NUPAD Diagnóstico situacional do Programa Nacional de Triagem Neonatal nos estados brasileiros: relatório técnico / Núcleo de Ações e Pesquisa em Apoio Diagnóstico - NUPAD. Belo Horizonte: NUPAD, 2013. 34 p.: il.

3. Carvalho DCSN, Macêdo TCC, Moreno M, et al. Evolução do programa de Triagem Neonatal em hospital de referência no Ceará: 11 anos de observação. Arq Bras Ciências da Saúde. 2017;42(3):143-6.

4. Oliveira EF, Souza AP. A importância da realização precoce do teste do pezinho: o papel do enfermeiro na orientação da Triagem Neonatal. Id Line Rev Psicol. 2017;11(35):361-78.

5. Botler J, Camacho LAB, da Cruz MM, George P. Neonatal screening - the challenge of an universal and effective coverage. Cien Saude Colet. 2010;15(2):493-508.

6. Mendes LC, Santos TT, Bringel FA. Evolução do programa de triagem neonatal no estado do Tocantins. Arq Bras Endocrinol Metabol. 2013;57(2):112-9.

7. Potter BK, Etchegary H, Nicholls SG et al. Education and Parental Involvement in Decision-Making About Newborn Screening : Understanding Goals to Clarify Content. J Genet Counsel. 2015;24:400-8.

8. Mesquita APHR, Marqui ABT de, Silva-Grecco RL, Balarin MAS. Profissionais de Unidades Básicas de Saúde sobre a triagem neonatal. Rev Ciências Médicas. 2017;26(1):1.

9. Servidoni MF, Gomez CCS, Marson FAL, et al. Teste do suor e fibrose cística: Panorama da realização do teste em centros públicos e privados do estado de São Paulo. J Bras Pneumol. 2017;43(2):121-8.

10. Barbosa CP, Griz SMS. Educação em saúde com vistas à triagem neonatal e audição: uma revisão integrativa. Rev CEFAC. 2014; 16(2):643-50.

11. Tluczek A, Orland KM, Nick SW, Brown RL. Newborn Screening: an appeal for improved parent education. J Perinat Neonat Nurs. 2009;23(4):326-34.

12. Temme R, Gruber A, Johnson M, et al. Assessment of parental understanding of positive newborn screening results and carrier status for cystic fibrosis with the use of a short educational video. J Genet Counsel. 2015;24:473-81.

13. Sabarense AP, Lima GO, Silva LML, Viana MB. Characterization of mortality in children with sickle cell disease diagnosed through the Newborn Screening Program. J Pediatr. 2015;91(3):242-7.

14. Botkin JR, Rothwell E, Anderson RA, Rose NC, et al. Prenatal education of parents about newborn screening and residual dried blood spots a randomized clinical trial. JAMA Pediatr. 2016;170(6):543-9.

15. Chudleigh J, Buckingham S, Dignan J, et al. Parents' Experiences of receiving the initial positive Newborn Screening (NBS) result for cystic fibrosis and sickle cell disease. J Genet Couns. 2016;25(6):1215-26.

16. Arduini GAO, Balarin MAS, Da Silva-Grecco RL, De Marqui ABT. Knowledge of puerperal mothers about the guthrie test. Rev Paul Pediatr. 2017;35(2):151-7.

17. Palmer SB, Bednarz SE, Dilaj KA, et al. Universal newborn hearing screening in midwifery education: a survey. J Midwifery Women's Heal. 2016;61(4):435-41.

18. Barbosa CP, Aires JB, Yasmin I, et al. Newborn and infant hearing health education for nursing professionals. Braz J Otorhinolaryngol. 2013;79(2):226-32.

19. Ryan DJ, Mikula EB, Germana S, et al. Screening for critical congenital heart disease in newborns. Adv. Neonatal Care. 2014;14(2):119-28.

20. Krishnan LA, Lawler B, Van Hyfte S. Parent educational materials regarding the newborn hearing screening process. Int J Pediatr Otorhinolaryngol. 2017;95:34-8.

21. Falkenberg MB, Mendes TPL, Moraes EP, et al. Educação em saúde e educação na saúde: conceitos e implicações para a saúde coletiva. Ciência \& Saúde Coletiva. 2014; 19(3):847-852.

22. Evans A, LeBlanc K, Bonhomme N, Shone SM, et al. A Newborn Screening Education Best Practices Framework: Development and Adoption. Int. J. Neonatal Screen. 2019;5(2):22. DOI: 10.3390/ijns5020022.

23. Pimentel MCR, Fiqueiredo N, Lima MLLT. Construção e validação do Modelo Lógico do Programa de Triagem Auditiva Neonatal. Rev CEFAC. 2020;22(4):e14019 